\title{
EFFECTS OF THE SYNAPTIC TRANSMISSION'S DYNAMICS ON POSSIBLE NEURAL CODES
}

\author{
László Orzó ${ }^{1}$, Elemér Lábos ${ }^{2}$ \\ ${ }^{1}$ Computer and Automation Research Institute Hungarian Academy of Sciences Analogical \& \\ Neural Computing Systems Laboratory H-1111 Budapest Kende u. 13-17, Hungary \\ email: orzo@sztaki.hu \\ ${ }^{2}$ United Research Organization of the Hungarian Academy of Sciences at Semmelweis Medical \\ University, Neurobiology Group 1450 Budapest, Tüzoltó u. 58. Hungary \\ email: labos@ana1.sote.hu
}

\begin{abstract}
To examine the effects of paired pulse facilitation, long term synaptic modifications, as well as spike frequency adaptation on neural signal transmission, a simple model was applied. This way various input-output properties of the model units were described. Particularly the transmission of the mean and the standard deviation of the simulated synaptic currents were studied. The results indicate that the transfer of the mean value of the membrane currents can not be described in terms of synaptic weights. So firing rate can hardly be an efficient neural code, especially for adaptive channels of the central nervous system (CNS).

On the contrary, the transfer of the standard deviation of synaptic currents behaves in accordance with the synaptic weights. So it is supported that standard deviation of the synaptic currents is a biological relevant subclass of the variation codes (see Perkel et al., 1968). It is discussed, how this code can be established and how it works.
\end{abstract}

Keywords: Neural code, Dynamics of synaptic transmission, Spike frequency adaptation, Synaptic plasticity;

\section{Introduction}

So far many details of the problem, how neurons communicate with each other, are not fully revealed (Perkel et al., 1968; Mitchison et al., 1990). Presumably different regions of nervous systems use special communication rules (e.g. Bialek et al., 1991). It is still an open question what the neural codes within the cerebral cortex are [Deuchars et al., 1995.].

To understand some aspects of neural information processing it seems to be fruitful to consider computation strategies used in various artificial neural network (ANN) models, not only findings of neuroscience.

In some ANN models the neuronal input is defined as the weighted sum of activity of the converging neurons. The change of these weights fundamentally determines the operation of the network.

In many ANN models, it is also implied that the firing rate transmits information between neurons and even its universal significance is claimed [Gerstner \& van Hemmen, 1992]. An overwhelming majority of experimental data demonstrates that the firing rate in fact 
changes for adequate stimulation [e.g. Richmond et al., 1990].

However, doubts arise if we consider the firing rate as a neural code at the higher levels of the CNS (e.g. in the neocortex):

- In the CNS, the neuronal firing level is usually low.

- At the same time, the coefficient of the variation of the inter-spikeintervals is high [Softky \& Koch, 1993].

- Besides, for example cortical pyramidal cells show a considerable spike-frequency adaptation.

It can be concluded that it takes a long time to measure the mean neuronal activity at low firing rate and high variability. During such a long period we have to take into account also the effects of spike-frequency adaptation. However, there is no doubt that the firing rate plays an essential role in the peripheral nervous system and in some central areas.

Other neural code candidates that emphasize particular kinds of neural response properties can not be excluded upon the above facts.

For example, it is claimed that precise timing and/or synchronization of action potentials convey information in the CNS [Singer, 1993]. In certain circumstances, the neurons show high precision of firing [Bair $\&$ Koch, 1996, Bialek et al., 1991].

- The precise interval code is obviously more efficient than the 'robust' rate code [Softky, 1994].

- High temporal precision of the neuronal action potential generation and its central role in signal processing are claimed [König et al., 1996].

- It is questioned, however, whether neurons in the CNS are capable of processing at this level of temporal accuracy [e.g. Shadlen \& Newsome, 1994].
In light of these concerns [Zador, 1998], in this paper, we aim to examine only the transmission of two "statistical" parameters of the neural activity. We try to specify the effects of the known properties of the synaptic transmission on the transfer of the mean and standard deviation of the synaptic currents.

\section{Model}

In the next sections phenomenological models of the spike frequency-adaptation and "dynamic redistribution of synaptic efficacy" are formulated. Makram and Tsodyks (1996) introduced this terminology for the case when the envelope curve of the averaged postsynaptic currents evoked by pulse sequences reaches a steady level. Furthermore, this static level depends only on the frequency of the presynaptic volley. Other parameters of the evoked postsynaptic currents, like initial amplitude and speed of decrease, depend also on the character of the synaptic modifications (e.g. LTP and LTD).

There are considerable simplifications in the present model. The following fundamental mechanisms were ignored:

- Detailed mechanisms of action potential generation were neglected.

- Neither the accurate time course of the evoked action potentials, nor the EPSPs' exact dendritic propagation was taken into account.

- We did not incorporate into this work such important phenomena, like burst response, pacemaker activity or even the effects of inhibitory neurons on the actual neural activity.

It is believed, however, that our principal purpose, the examination of the neural information transfer, can be significantly promoted regardless of the above factors. 


\subsection{Dynamic redistribution of the synaptic efficacy}

There are relatively good models of the dynamic redistribution of the synaptic efficacy (Tsodyks, 1997; Abbot et. al., 1997). However, these models simulate only the mean value of the evoked postsynaptic currents (potentials). As our intention was to compute the standard deviation of the evoked postsynaptic currents as well, here we incorporated a binomial model of the synaptic release (Burnod \& Korn, 1989) and a simplified dynamics of the depletion and refill of transmitter vesicles in this simulation.

$$
\begin{aligned}
& \text { For } i=1 \text { to } N \\
& \quad\left\{\text { If } \quad R_{i, n}=1\right. \text { then } \\
& \quad \text { with p prob. }\left\{S_{n}=S_{n}+1 ;\right. \\
& \left.\quad R_{i, n}=0 ; q_{i}=0 ;\right\} ; \\
& \quad\} ; \\
& q_{i}=q_{i}+\left(1-q_{i}\right)\left(1-\exp \left(\frac{-I S I}{T}\right)\right) \text {; } \\
& \text { if } \quad R_{i, n}=0 \text { then } \\
& \text { with } q_{i} \text { prob. } R_{i, n+1}=1 ;
\end{aligned}
$$

The $\mathrm{R}_{\mathrm{i}, \mathrm{n}}$ variable corresponds to the available resource at the $\mathrm{i}^{\text {th }}$ release site after the $\mathrm{n}$-th emitted action potential. The $\mathrm{N}$ variable specifies the number of release sites between pre- and postsynaptic neurons. If a release site is active, i.e. it is not empty $\left(\mathrm{R}_{\mathrm{i}, \mathrm{n}}=1\right)$, it can release a quantum of transmitter for an incoming action potential with some probability $(p)$. This probability corresponds to the synaptic strength and changes according to the long-term synaptic modifications (increases for LTP and decreases for LTD) (Stevens \& Wang, 1994; Volgushev et al., 1995). The size of the quanta was taken to be equal for all the release sites. After a release the site becomes inactive, depleted $\left(\mathrm{R}_{\mathrm{i}, \mathrm{n}}=0\right)$ and its recovery takes a considerable time. The instantaneous synaptic efficacy (S) is updated in this model every time the presynaptic cell releases an action potential. Its value accounts for the number of actually released quanta, which depends on, as we have mentioned above, the transmission probability (p) and the number of instantaneously active release sites $\left(\mathrm{R}_{\mathrm{i}, \mathrm{n}}\right)$. The subscript $\mathrm{n}$ encounters action potentials.

In this model, recovery was considered as a stochastic process (2), and only the release site's recovery or refill probability is given $\left(\mathrm{q}_{\mathrm{i}}\right)$. The evolution of this variable is the function of two parameters, $\mathrm{T}$ and ISI, which correspond to the recovery time constant and inter-spike-interval respectively.

This simple simulation can assure that at low rate firing, the synaptic efficacy reaches the $\mathrm{Np}$ average value (Burnod \& Korn, 1989).

The model reproduces several findings: The amplitudes of the simulated EPSPs depend on the previous cell activity. If a train of action potentials evokes postsynaptic potentials, the mean peak amplitudes of the consecutive EPSPs decrease until they reach a stable, static value which depends only on the train's inter spike intervals.

To demonstrate the capability of the simulation we depict the model's response for spike sequences with different interspike-intervals (Figure 1).

Some parameter values were chosen for proper reproduction of the measured data. Others were fit into experimental facts $(\mathrm{N}=20$; Thomson, 1993b, 1995: $\mathrm{N}>10)$. The recovery time constant was set to a low value because of the measured slow recovery of the synaptic efficacy (Tsodyks \& Makram, 1997 $) \quad(T=0.001)$. The effects of the LTP and LTD are considered as an increase and decrease of the (p), the release probability value $(0.3-0.9)$. 


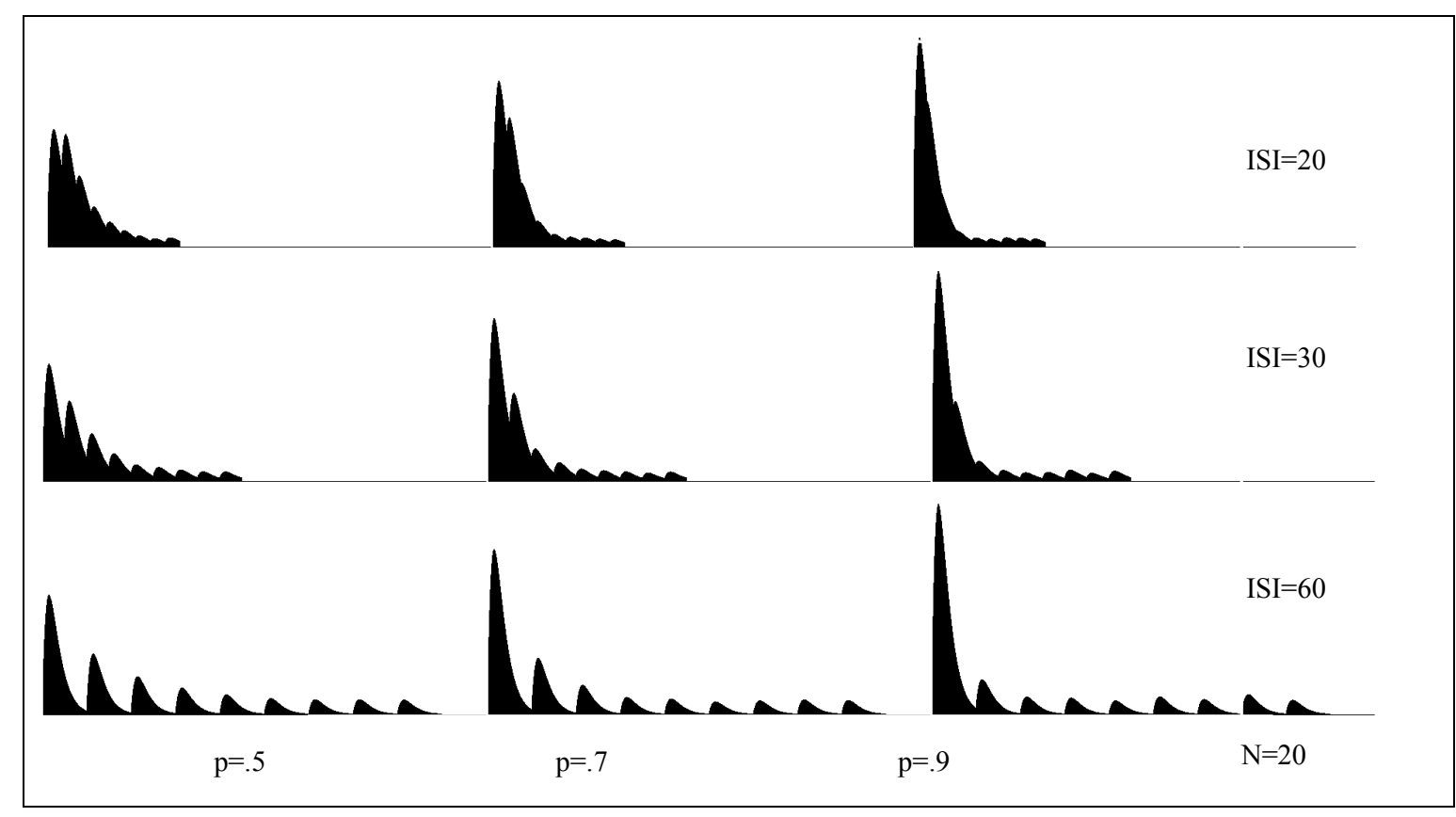

Figure 1. If a train of action potentials evoke postsynaptic potentials, the average amplitudes of the consecutive EPSPs decrease until they reach a stable value that depends on Inter-SpikeIntervals (ISI). The probability of synaptic transmission (PR), however, determines the initial value and decline of consecutive postsynaptic potentials average size in a spike train. (Actual details of the model's EPSP generation are discussed later)

\subsection{Generating the presynaptic spike train and its adaptation}

To estimate the effects of the synaptic transmission dynamics on the neural information transfer, action potential sequence was required. Since this kind of experimental data was not available, simple simulation was applied.

For the simulation of the neural firing patterns, a simple 'integrate and fire' neuron model with spike frequency adaptation was introduced (Sah, 1996). We could use elaborated and biologically more faithful multi-compartmental Hodgkin-Huxley $(\mathrm{HH})$ type of models as well, but the generality of this approach, due to the big amount of parameters is questionable. Even the big variability of inter-spike-intervals is hard to establish by $\mathrm{HH}$ models.

The model's input was taken to be a normally distributed random variable. It corresponds to the sum of presynaptic action potentials weighted by the synaptic strengths. Assumption of its normal distribution is supported by the Central Limit Theorem (CLT), since the number of converging synapses is high. It is supposed that the firing of the presynaptic neurons is not correlated. A modest level of the correlation, however, does not considerably confine the applicability of this model. If the neurons receive correlated inputs it will be followed by the neural output. In this work, however, we expect that there is no considerable correlation between the neuron's activity.

To simulate synaptic currents second order linear process are applied. If the simulated membrane potential reaches a threshold, an action potential is released. A quickly inactivating, hyperpolarizing current restores the membrane potential (not shown). Spike frequency adaptation was simulated by a slowly inactivating afterhyperpolarization current. This current follows all the released action potentials and 
decreases the model neuron's membrane potential for a prolonged period of time. Our model can be specified by the following iterations:

$$
\begin{aligned}
& T_{n+1}=a T_{n}+X \\
& C_{n+1}=b C_{n}+T_{n} \\
& A_{n+1}=c A_{n}+\psi\left(U_{n}\right) \\
& U_{n+1}=d U_{n}+C_{n}-A_{n}
\end{aligned}
$$

(Where: $a=0.9 ; b=0.9 ; c=0.9995 ; d=0.4$; $\Theta=5 ; \psi(\mathrm{U})=0.3(\operatorname{sign}(\mathrm{U}-\Theta)+1) ; \mathrm{X}=$ Input $)$

The ' $T$ ' variable corresponds to the is according to the instantaneous synaptic efficacy, which was simulated in the previous section.

The ' $O C$ corresponds to the postsynaptic current evoked by the ' $O T$ '.

\section{Modeled neurons input-output relation}

Having a simple model of the spiking activity and that of the dynamic changes of the synaptic efficacy, we can combine them. A sample of simulated neural activities is displayed in Figure 2.

The transmission of two parameters of the simulated neural activities was

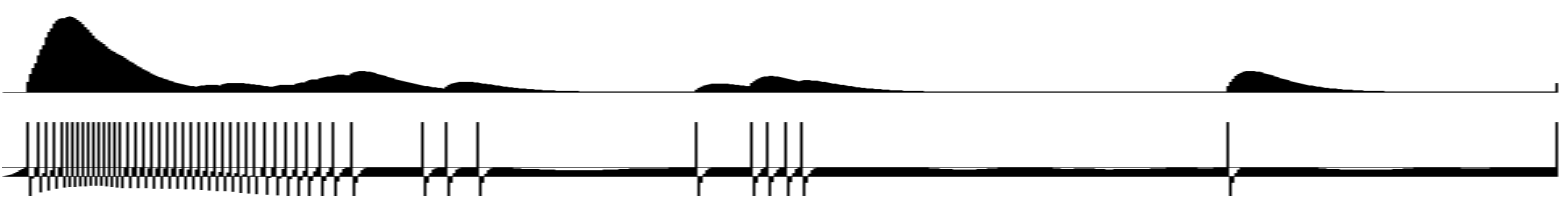

Figure 2. Effects of adaptation and redistribution of synaptic efficacy on postsynaptic currents. The lower line indicates the simulated presynaptic neuron's membrane potential. The upper line shows postsynaptic currents elicited by modeled presynaptic activity.

summed quantity of transmitters reaching the model neuron, produced by the convergent synaptic inputs.

The ' $C$ ' is according to the integrated synaptic currents, is evoked by ' $T$ ';

The ' $A$ ' corresponds to the afterhyperpolarization current;

The ' $n$ ' index indicates discrete time steps (note that this n plays different role in (1) and (3)) ;

To obtain the comparable input and output variables, the evoked postsynaptic currents are simulated by the following way:

$$
\begin{aligned}
& O T_{n+1}=a O T_{n}+S \\
& O C_{n+1}=b O C_{n}+O T_{n}
\end{aligned}
$$

Parameters were chosen to be the same as in (3)

The 'OT' corresponds to the amount of transmitter released postsynaptically;

The ' $S$ ' is the amount of transmitter evoked by action potentials. This parameter examined: the mean and standard deviation of membrane currents. These two parameters can fully describe the neuron's summed input.

Only the synaptic currents of the input and output were compared because in the membrane potential afterhyperpolarization and other currents are involved as well.

Our result indicates that the average output current is proportional to the mean of the input current and its dependence on the input current's deviation is inferior. While, the standard deviation of the output current is depending on both the average and deviation of the input current. Since the coefficient of variation of the output current occurred to be high, its measurement is cumbersome.

Transfer properties of the combined model are displayed in Figure 3. 

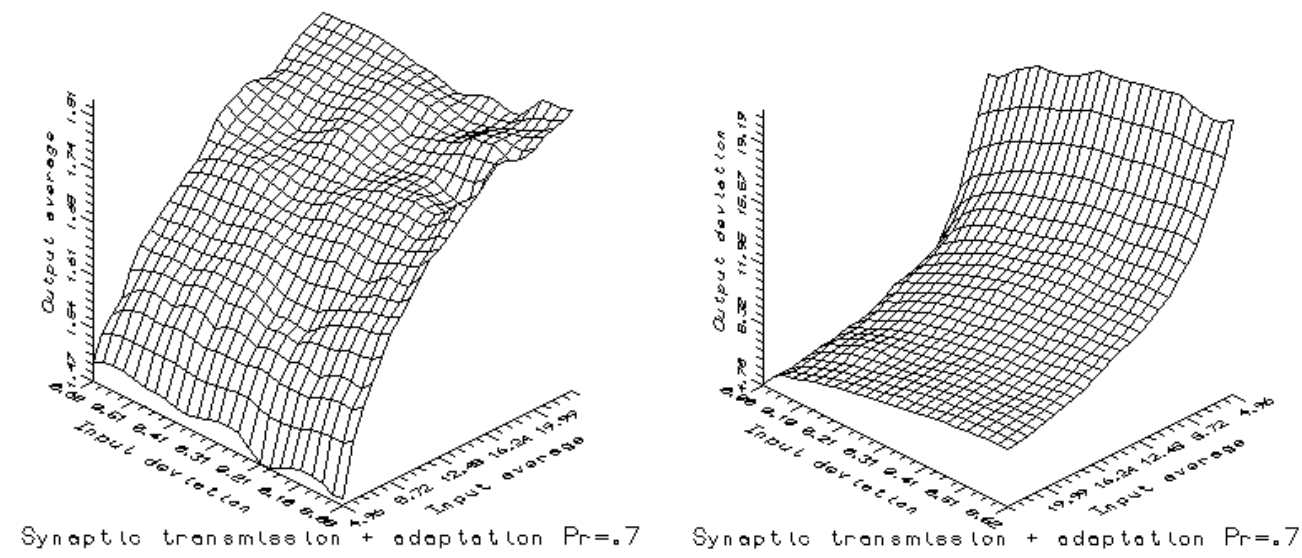

Figure 3. The model neuron's average evoked postsynaptic (output) current (A) and its deviation (B) as functions of average presynaptic (input) current and deviation.

Our simulation shows that there is an almost linear relationship between the average output and input currents.

It is a striking behavior of the inputoutput function that there is no enhancement in the slope and only a shift of the mean value at any input current level. This means that the change of the synaptic transmission is not manifested in an increase of the synaptic weight but only in some kind of shift of the threshold (Figure 4)!

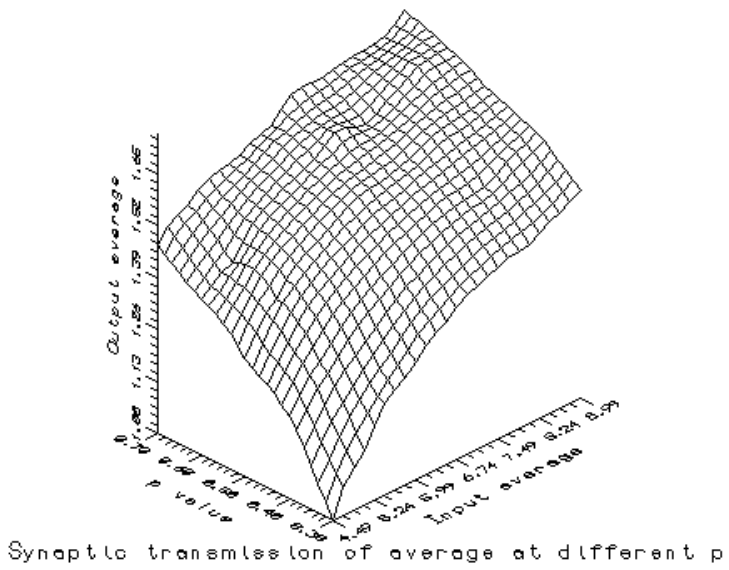

Figure 4. The average evoked postsynaptic current as a function of average input current and transmission probability.

The observable changes point just in the opposite direction than expected. Minimal value of the simulated input current generates about $3 \mathrm{~Hz}$ activity, which approaches the cortical background activity.

The other examined parameter in Fig. 3 behaves in quite a different way. Transfer properties of the standard deviation of synaptic currents at various levels of the transmission probability and at a fixed value of the average input current are depicted in Figure 5.

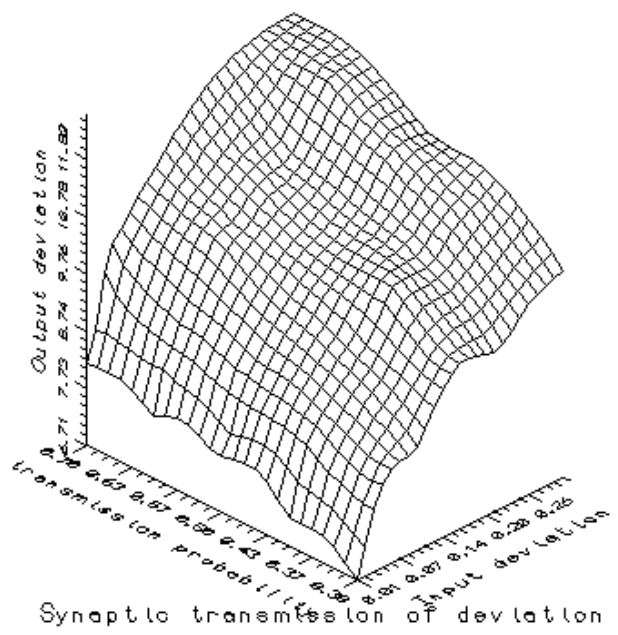

Figure 5. Dependence of the evoked postsynaptyic current's standard deviation on the input current deviation and transmission probability.

It can be seen that the dynamic range of the deviation transfer is expanding as the transmission probability is increasing (corresponding to the LTP). A change in the 
transmission probability leads to a simultaneous change in the slope of deviation input-output relation. Metaphorically speaking, the slope of this function can be considered as a new type of "synaptic weight". This finding indicates that transmission of deviation behaves according to the synaptic weights in some neural network models.

\section{Conclusions and Discussion}

Adaptation processes confine the signal parameters that can be transmitted. Furthermore, they can provide mechanism of the establishment and transmission of these parameters.

There are considerable limits of the applicability of firing rate code, as we discussed it in the introduction. Our results indicate further doubts: Long-term synaptic modifications do not change the synaptic weights appropriately, but shift only the apparent threshold.

Probabilistic properties of the synaptic release and transmission, however, make other hypothetical codes, in which the fine temporal structure of the spike trains is supposed to transmit information, also unlikely.

We have found that another investigated variable, the standard deviation of synaptic currents, can convey information and can be regarded as a neural code within CNS. This variable is determined straightforwardly just like the average synaptic currents. As it has been mentioned above, the slope of the deviation input-output relation behaves like a "pseudo synaptic weight" and appropriately reacts to the LTP and LTD.

From theoretical point of view it seems to be useful if we can provide mechanism that can solve (Orzó, 1993) the transformation of the frequency code to this code, also the neural transmission using this type of coding, and finally some type of translate this code to firing rate. Our results show that the model neuron transfer properties make these transformations possible:

There is an approximately linear relationship between the output deviation and input average at any level of the transmission probability (corresponding to LTP-LTD). Namely, the higher the presynaptic current's averaged amplitude the lower the evoked postsynaptic current's deviation will be (Figure. 3.). Certain experimental data (Tsodyks, 1997; Softky and Koch, 1993) indicate the possibility and reality of such a conversion.

Computation based on such a deviation code may occur in neural system, like in the case of the firing rate code in some ANN models. The corresponding variables follow similar rules.

Conversion from the suggested deviation to the firing rate code can be achieved by using paired pulse facilitation. Neurons with high membrane current (and so firing rate) variability generate much higher average EPSCs than neurons with low variability of the firing rate, if they are capable of paired pulse facilitation. Astonishingly, this property is observed in deep layer cortical pyramidal neurons (Thomson, 1993a,b).

Additionally, accurate measurement of the standard deviation can be much easier than that of the mean, depending on their respective distribution. Due to its special definition, the sensitivity of this code against various noise and propagation uncertainties is small.

Acetylcholine (Tsodyks, 1997) and other neuromodulators decrease the probability of transmitter release (LTD) and so they are able to tune the dynamics of the whole neural network.

Our results can be validated by the simulation of the evoked postsynaptic currents for measured spike sequences originating from relevant experiments. 


\section{References}

Abbot, L.F., Varela, J.A., Kamal Sen, Nelson S.B. Synaptic depression and Cortical gain control. Science, 275:220-224, 1997.

Bair, W., Koch, C., Temporal precision of spike trains in extrastriate cortex of the behaving macaque monkey. Neural Computation, 8: 1185-202, 1996

W. Bialek, F. Rieke, R.R. de Ruyter van Steveninck, D. Warland. Reading a Neural Code. Science, 252:1854-1857, 1991.

Y. Burnod, H. Korn. Consequences of stochastic release of neurotransmitters for network computation in the central nervous system. Proc. Natl. Acad. Sci. USA., 86:352-356, 1989.

J. Deuchars, D.C. West, D. Ferster, N. Spruston. Cracking the Neural Code. Science, 270: 756757, 1995.

W. Gerstner, J.L. van Hemmen. Universality in neural networks: the importance of the 'mean firing rate' . Biol. Cybern. 67:195-205, 1992.

König P., Engel A., Singer W., 1996. Integrator or coincidence detector? The role of the cortical neuron revisited. TINS. 19(4), 130-137.

Markram H., Tsodyks M., 1996. Redistribution of synaptic efficacy between neocortical pyramidal neurons. Nature 382, 807-810.

Mitchison G., Miall C., 1990. The Enigma of cortical code. TINS. 13(2), 41-43.

Orzó L., 1993. Deviation code is a prospective candidate of the communication between adapting neurons. Neurobiology 1(3), 223234.

Perkel H., Bullock T.H., 1968. Neural Coding. Neuroscience Research Program Bulletin 6, 221-344.

Richmond B.J., Optican L.M., 1990. Temporal Encoding of two-dimensional Patterns by Single Units in Primate Primary Visual Cortex II. Information Transmission. Journal of Neurobiology 64(2), 370-380.

Sah P., 1996. $\mathrm{Ca}^{++}$-activated $\mathrm{K}^{+}$currents in neurons: types, physiological roles and modulation. Trends in neuroscience 19(4), 150-154.

Singer W., 1993. Synchronization of cortical activity, and its putative role in information processing and learning. Annual Review of Physiology 55, 349-374.
Shadlen M.N., Newsome W.T., 1995. Is there a signal in the noise? Current Opinion in Neurobiology 5, 248-250.

Softky W., Koch C., 1993. The Highly Irregular Firing of Cortical Cells Is Inconsistent with Temporal Integration of Random EPSPs. J. of Neuroscience 13(1), 334-350,.

Softky, W.R., 1995. Simple codes versus efficient codes. Current Opinion in Neurobiology 5, 239-247.

Stevens, C.F., Wang, Y., 1994. Changes in reliability of synaptic function as a mechanism for plasticity. Nature 371, 704-707.

Thomson, A.M., West, D.C., 1993.a, Fluctuations in pyramid-pyramid excitatory postsynaptic potentials modified by presynaptic firing pattern and postsynaptic membrane potential using paired intracellular recordings in rat neocortex. Neuroscience 54(2), 329-360.

Thomson, A.M., Deuchars, J., West, D.C., 1993.b, Large, Deep Layer Pyramid-Pyramid Single Axon EPSPs in Slices of Rat Motor Cortex Display Paired Pulse and FrequencyDependent Depression, Mediated Presynaptically and Self-Facilitation, Mediated Postsynaptically. Journal of Neurophysiology 70, 2354-2369.

Thomson, A.M., West, D.C., Deuchars, J., 1995. Properties of single axon excitatory postsynaptic potentials elicited in spiny interneurons by action potentials in pyramidal neurons in slices of rat neocortex. Neuroscience 69(3), 727-738.

Tsodyks, M.V., Markram, H., 1997. The neural code between neocortical pyramidal neurons depends on neurotransmitter release probability. PNAS 94, 719-723.

Volgushev, M., Voronin, L.L., Chistiakova, M., Artola, A., Singer, W. 1995. All-or-none Excitatory Postsynaptic Potentials in the Rat Visual Cortex. European Journal of Neuroscience 7, 1751-1760.

A. Zador (1998) The Impact of Synaptic unreliability on the Information Transmitted by Spiking Neurons. Journal of Neurophysiology (in press) 